\title{
Biodegradable Topical Nanogels in the Treatment of Skin and Superficial Tumors
}

\author{
Sushil K Kashaw ${ }^{1,2 *}$ and Arun K Iyer ${ }^{1,3 *}$ \\ ${ }^{1}$ Department of Pharmaceutical Sciences, Wayne State University, USA \\ ${ }^{2}$ Department of Pharmaceutical Sciences, Dr Harisingh Gour University, India \\ ${ }^{3}$ Molecular Therapeutics Program, Wayne State University, USA
}

Submission: January 16, 2017; Published: February 07, 2017

*Corresponding author: Arun K Iyer, Department of Pharmaceutical Sciences, Eugene Applebaum College of Pharmacy and Health Sciences, 259 Mack Ave, Room 3601, Wayne State University, Detroit, MI 48201, USA, Tel: 313-577-5875; Fax: 313-577-2033; Email: arun.iyer@wayne.edu

\begin{abstract}
Nanogels are nano-sized three-dimensional polymer networks engineered by physical or chemical cross-linking of individual polymer chains loaded with drugs and nucleotides that swells in a good solvent
\end{abstract}

Keywords: Biodegradable nanogel; Cross-linked polymer; Cancer; Chitosan; Nanomedicine; Nanotechnology

\section{Introduction}

Nanomedicine and nanotechnology is heralded to play a pivotal role in the management of a several disease conditions with implication in prevention, diagnosis and effective treatment of patients. In the scenario of cancer, the impact of nanotechnology is proving to have a profound positive influence. By taking advantage of the unique features of nanoparticles and nanotechnology based biomaterials, new precision guided arsenals are being developed infighting the war against cancer. In this regard, nanogels are emerging as a one of the promising vectors for drug delivery and they have been largely screened for various cancers in the last few decades. However, the material toxicity and tissue selectivity are considered as major barriers for their successful translation [1]. Addressing the toxicity issues related to polymeric and inorganic/ organic nanocarriers has become critical for bringing such new agentsintotheclinical realm. Along theselines, theresearch fraternity has focused their efforts on the use of biodegradable materials for the fabrication of nanogels and nanoparticles. Biodegradable nanogels exhibiting favorable particle size entrapping bioactive such as anticancer drug and gene payload, when administered topically or transdermally can reach the desired site and deliver the drug payload (for instance, melanoma). The degradation products of the polymers are amenable for elimination via various biological mechanisms taking place within the body [2]. The biodegradable nature of the carries thus does not necessitate any specific care in its use and does not pose any safety concern while in use. This approach ensures delivery of desired dose of cytotoxic agents to the targeted site effectively, whereas the use of biologically safe or degradable components eliminates the unwanted adverse reaction caused by the carrier system. In the present scenario, a wide variety of nanocarriers with spatial and temporal control on the properties are being designed and developed using polymeric biomaterials, Dendrimer and liposome's, that can be loaded with various therapeutic agents as well as conjugated with targeting ligands for more specific targeting [3].

Biodegradable Nanogel Synthesis Constituting $\mathrm{PH}$ Responsive Characteristic

Biodegradable nanogels are currently emerging as one of the potential carriers for treating diseases such as cancers, which can enhance therapeutic efficacy of drugs or bioactive at the target site (such as cancer osuleisions) and reduce its adverse toxicity to non-target organs and tissues. Nanogels can be synthesized by various methods and they generally exhibit a three-dimensional network of "cage" in which the active molecule or drug is entrapped. These nanogel networks can be made to possess stimuli sensitive characteristics such as $\mathrm{pH}$, enzymatic or temperature responsiveness, that can, "on demand" either trigger the collapse or degradation of the matrix to result in drug release (at the site of action), based on the requirement of the nanogel system. The biodegradable nature of the polymers in conjunction with stimuli 


\section{Global Journal of Nanomedicine}

responsive nature (such as $\mathrm{pH}$ or enzymatic responsiveness) can enhance safe and effective drug delivery with reduced off-target effects [4].

In addition, the nanogel scan be made to possess an ability to carry large payload of drugs and allows for sustained release of cytotoxic agents at the site of action (such as cancerous lesions). The conventional nanocarriers made of or constituting inorganic or organic (non-degradable) constructs such as metals, metals-ion, and chelates can elicit unwanted adverse reaction in the biological setting and can cause toxicities. Non-biodegradable carriers also have a tendency to reside in the body for longer time intervals leading to acute toxicities; however nanogels constructed using biodegradable polymers or biologically derived agents are devoid of such toxicity issues. Thus, biodegradable nanogel based targeted drug delivery offers efficacious and site specific delivery providing an opportunity for better management of the disease condition within a reasonable time to ensure good patient compliance and safety. Biodegradable topical nanogels can also be suitable for all types of cancer patients (sensitive, aged or young adults or children). More importantly, the sustained release nature can help avoid frequent dosing and improve patient compliance. In terms of economy of scale, the nanogels can be produced on an industrial scale and the raw materials can be affordable, providing high costto-benefit ratio as well [5].

Drug development necessarily requires trade-offs among desired drug features, toxicity and patient compliance; but for the treatment of cancer, in the developing world, the provision of affordability, safety, robustness of the nanocarriers are highly demanding. Although some researchers have reported that the nanocarriers or novel drug delivery system are still under development and are not readily accepted as an alternative mode of therapy due to their safety concerns, biodegradable nanogels have tremendous opportunity for the treatment of diseases such as cancers with high therapeutic efficiency with reduced safety concerns [6]. In this regard, the delivery of drug also depends on several factors such as biocompatibility, tissue response, patient profile, age, severity of the disease, which directly or indirectly influences the treatment pattern and dosing regimen. One way to overcome these challenges is to design biodegradable materials or drug carriers which possess potential therapeutic effects with less toxicity or to fabricate the dosing agents in a manner that they are biologically safe/inert or biodegradable, or that can be cleared out of the body efficiently.

In this regard, topical or transdermal drug carriers should ideally possess molecules size in the range of 100-500 Da, for efficient penetration and transport of material, as well as clearance after the treatment window. A wide variety of biodegradable materials are currently emerging which show great potential as carriers for drug delivery [7]. In one such effort, we recently employed the ionic interaction between the pendant cationic amino groups present on the biodegradable chitosan moiety with the anionic phosphate group of TPP (sodium tri poly phosphate) or glycerol phosphate which lead to the efficient chemical cross linking leading to the formation of biodegradable nanogel network. In this study, we found that the natural origin chitosan polymer and glycerol phosphate or TPP could form hydrogels with favorable drug loading properties with excellent biodegradability and demonstrated rapid clearance as the bi-product of various enzymatic reactions in body [8]. Advanced studies suggest that the biodegradable nanogel synthesize by the ion gelation method with the aid of Pluronic-127 exhibits formation of monomolecular micelles at low concentration whereas it exhibits multi-molecular aggregates at higher concentration involving hydrophobic central core containing hydrophilic poly oxyethylene chains in the corona.

\section{Functionality \& Benefits of Biodegradable Nanogel}

Nanogel-based therapeutics has been successfully developed for disease diagnosis, imaging and therapy. Specifically, nanogels can enhance the therapeutic efficiency and eliminate the adverse effect and toxicity of the therapeutic agents when delivered topically or transdermally. Typically, when biodegradable nanogel is administered topically, it exhibits the ionic interaction with $\mathrm{pH} /$ stimuli-triggered release of the drug at targeted site showing excellent release kinetics with quick degradation of polymer networks. However, such functionality should be designed with low cost synthesis with a robust nanogel system. In some cases the cost of production of nanogel with additional stimuli-responsive features may impact the commercial production [9]. However, additional modification like surface coating, ligand tailoring makes the nanogels more attractive for treating a varieties of ailments. In this regard, chitosan possesses favorable cationic nature and slight acidic pH range of 5.0-6.0 units and the tumor environment shows negative charge on its cell membrane due to accumulation of lactic acid by elevated use of oxygen by cancer cell and exhibits $\mathrm{pH}$ 5.5-6.5. This ionic interaction and $\mathrm{pH}$ responsive characteristic makes nanogel system a ideal for targeted cancer cell killing [10]. This strategy for cancer treatment allows for development of numerous drug delivery systems with various payloads to cater to several types of skin or superficial cancers. In our case, we decreased the concentration of polymer and cross-linker to improve their biocompatibility, and then introduced Pluronic 127 as a hydrophilic copolymer which forms monomolecular micelles at low concentration. Since the whole systems possess a $\mathrm{pH}$ range of 5.0-6.5 units the extra refinement in adjustment of the $\mathrm{pH}$ was bypassed resulting in reducing the costs greatly.

The stoichiometry of all components were well controlled and the nanogels were placed at ambient temperature and chemical and biological conditions so that further assessments by employing various chemical and analytical evaluations guaranteed the consistency of components in the formulation, and assured reproducible pharmacokinetic behavior of the therapeutic agents in the body [11]. The core-shell particles loaded with cytotoxic agents revealed utmost advantage of ultrafine size between 200$300 \mathrm{~nm}$ which mimics the skin environment for the effective penetration when administered topically. Such nanogel delivery 
system is not limited to the nanoparticles, but can also be utilized for multi vesicular carriers such as liposome, niosomes, and phytosomes. Therefore, other drugs such as antimicrobial and antifungal agents can also be incorporated into the nanogel matrix to evaluate combination therapies for various disease conditions. Importantly, the nanogel particles can be rapidly cleared out from the body after post treatments via renal excretion and/or enzymatic degradation to reduce the unwanted toxicity. On the other hand, use of biodegradable nanogel vector exhibits sustained and extended release features for favorable long term response [12].

\section{Outlook}

The future potentials for biodegradable nanogels offers great promises and provides novel opportunities for researchers for application in drug delivery against wide varieties of disease and disorders. By modification of nanogel system and fine tuning its compositing such as polymer type, molecular weight, and cross-linking density, we can attain optimal drug loading in the biodegradable nanogels. Furthermore, additional functional features such as imaging probes and targeting moieties can be easily anchored onto nanogels for exploring their targeted the ranostic potentials. We believe that the optimal nano-sized nature with multifunctional biodegradable 3-dimensional polymer architecture, with stimuli-responsive features, would make such agents highly attractive for topical targeted cancer therapy with reduced toxicity burden to patients.

\section{Financial \& Competing Interest Disclosure}

This work was supported by the Department of Pharmaceutical Sciences, Dr. Harisingh Gour University, Sagar, MP, India. SKKacknowledges the University Grants Commission, New Delhi for the award of Raman Postdoctoral Fellowship.

\section{References}

1. Oh NM, Oh KT, Baik HJ, Lee BR, Lee AH, et al. (2010) A self- organized 3diethylaminopropyl- bearing glycol chitosan nanogel for tumor acidic pH targeting: in vitro evaluation. Colloids Surf B Biointerfaces 78(1): 120-126.

2. Motoi Oishi, Naoki Miyagawa, Takeshi Sakura, Yukio Nagasaki (2007)
pH- responsive PEGylated nanogel containing platinum nanoparticles: Application to on-off regulation of catalytic activity for reactive oxygen species. Reactive and Functional Polymers 67(7): 662-668.

3. Owens DE, Peppas NA (2006) Opsonization, biodistribution, and pharmacokinetics of polymeric nanoparticles. Int J Pharm 307(1): 93102.

4. Panagi Z, Beletsi A, Evangelatos G, Livaniou E, Ithakissios DS, et al. (2011) Effect of dose on the biodistribution and pharmacokinetics of PLGA and PLGA- mPEG nanoparticles. Int J Pharm 221(1- 2): 143-152.

5. Mohamed F, van der Walle CF (2008) Engineering biodegradable polyester particles with specific drug targeting and drug release properties. J Pharm Sci 97(1): 71-87.

6. Mohandas KM, Desai DC (1999) Epidemiology of digestive tract cancers in India. V. Large and small bowel. Indian J Gastroenterol 18(3): 118-121.

7. Mason TG, Wilking JN, Meleson K, Chang CB, Graves SM (2006) Nanoemulsions: formation, structure and physical properties. J Phys: Condens Matter 18: 635-666.

8. Mc Cormick DL, Moon RC (1986) Antipromotional activity of dietary $\mathrm{N}$ - (4- hydroxyphenyl) retinamide in two- stage skin tumorigenesis in CD- 1 and SENCAR mice. Cancer Lett 31(2): 133-138.

9. Luo X, Xie C, Wang H, Liu C, Yan S, et al. (2012) Antitumor activities of emulsion electrospun fibers with core loading of hydroxyl camptothecin via intratumoral implantation. Int J Pharm 425(1-2): 19- 28.

10. Maeda H, Wu J, Sawa T, Matsumura Y, Hori K (2000) Tumor vascular permeability and the EPR effect in macromolecular therapeutics: a review. J Control Release 65(1- 2): 271-284.

11. Lee J, Yoo HS (2008) Pluronic decorated nanogels with temperature responsive volume transitions, cytotoxicities and transfection efficiencies. Eur J Pharm Biopharm 70(2): 506-513.

12. Kayal S, Ramanujan RV (2010) Doxorubicin loaded PVA coated iron oxide nanoparticles for targeted drug delivery. Materials Science and Engineering: C 30(3): 484-490.
This work is licensed under Creative

Commons Attribution 4.0 License

DOI: $10.19080 / G J N .2017 .01 .555556$
Your next submission with JuniperPublishers will reach you the below assets

- Quality Editorial service

- Swift Peer Review

- Reprints availability

- E-prints Service

- Manuscript Podcast for convenient understanding

- Global attainment for your research

- Manuscript accessibility in different formats

( Pdf, E-pub, Full Text, Audio)

- Unceasing customer service

Track the below URL for one-step submission

https://juniperpublishers.com/submit-manuscript.php 